\title{
Medir el uso del espacio público urbano seguro ${ }^{1}$
}

\author{
Measure the Use of Safe Urban Public Space
}

Medir o uso de espaços urbanos mais seguros

\author{
Sabina Cárdenas O'Byrne ${ }^{2}$ \\ Profesora e investigadora Pontificia Universidad Javeriana Cali, Colombia \\ sabinac@javerianacali.edu.co
}

Recibido: 30/01/2017

Aprobado: $24 / 05 / 2017$

\begin{abstract}
Resumen
El artículo aborda la medición del uso del espacio público como estrategia para mejorar la actitud frente a la seguridad, de tal manera que dicha seguridad no suponga una barrera para la revaloración de barrios. Para ello, se realizó una revisión bibliográfica en cuatro aspectos: la noción de barrio y el miedo como operador de la seguridad, la seguridad como agente de decisión para el uso del espacio, los mecanismos para medir espacio seguro, y las prácticas espaciales cotidianas. Esta revisión bibliográfica se pone en diálogo con una investigación denominada Ciudad Vital (Cárdenas, 2016), realizada en las ciudades colombianas de Palmira (2014) y Cali (2016). Se trata de generar alternativas para sumarse a las inquietudes de los autores consultados acerca de la medición del espacio público seguro y explorar una herramienta para el diseño urbano en términos de uso.
\end{abstract}

Palabras clave: medición; diseño urbano; observación; prevención del crimen; cambio de actitud.

\begin{abstract}
The article addresses the measurement of the use of public space as a strategy to improve the attitude towards security, in such a way that such security does not constitute a barrier to the revaluation of neighborhoods. For this, a bibliographic review was carried out in four aspects: the notion of neighborhood and fear as a security operator, the safety as a decision agent for the use of space, the mechanisms to measure safe space, and the daily spatial practices. This bibliographical review dialogues with a research called Ciudad Vital (Cárdenas, 2016), carried

\footnotetext{
${ }^{1}$ Artículo de revisión bibliográfica que busca aportar a la reflexión sobre el espacio público seguro de la investigación terminada titulada "Ciudad vital: una propuesta de espacios públicos urbanos seguros y vitales para Cali, Colombia" (02010094), financiada con recursos de la convocatoria interna de la Pontificia Universidad Javeriana (Cali, Colombia) para el periodo enero-diciembre de 2016. La reflexión también forma parte de la ponencia "El uso del espacio público urbano y la actitud frente a la seguridad: una metodología”, durante el XII Seminario de Investigación Urbana y Regional de la Asociación Colombiana de Investigadores Urbano Regionales (ACIUR), 2016, donde se mostraron los resultados en Palmira.

${ }^{2}$ Doctora en Arquitectura.
} 
out in the Colombian cities of Palmira (2014) and Cali (2016). It is about generating alternatives to join the concerns of the consulted authors regarding the measurement of safe public space and to explore a tool for urban design in terms of use.

Keywords: measurement; urban design; observation; crime prevention; change of attitude.

\section{Resumo}

Este artigo aborda a medição do uso do espaço público como estratégia para melhorar a atitude perante a segurança, toda vez que ela não suponha uma barreira para a revalorização dos bairros. Por tanto, se realizou uma revisão bibliográfica a partir de quatro aspectos: a noção de bairro e o medo como operador de segurança; a segurança como agente de decisão para o uso do espaço; os mecanismos para medir espaço seguro; e as práticas espaciais cotidianas. Essa revisão faz um dialogo com uma pesquisa denominada "Ciudad Vital (Cárdenas, 2016), feita nas cidades colombianas de Palmira (2014) e Cali (2016). Trata-se de gerar alternativas que se aderem às inquietações dos autores consultados, quem indagam na medição do espaço público seguro e exploram uma ferramenta para o desenho urbano em termos do uso.

Palavras-chave: medição; projeto urbanístico; observação; prevenção do crime; mudança de atitude.

\section{Introducción}

El barrio es fundamental para reconstruir la ciudad, y es en esta escala local que se resuelven los múltiples, graves y complejos problemas de las grandes ciudades. Esta escala es adecuadamente pequeña como para mantener una red social densa, que fomente, consolide y fortalezca la cohesión social entre moradores (Alguacil, 2008). La escala intermedia de los barrios ayuda a reducir la brecha entre las esferas pública y privada, al estar ubicada en su intersección (Madanipour, 2003).

En el barrio se da el tránsito entre el ser individual y el ser social, y el espacio público urbano opera como bisagra entre lo puramente familiar y las primeras relaciones amorosas, contactos deportivos y manifestaciones políticas del individuo que conforma su ser social (Buraglia, 1998). De acuerdo con este postulado, se requiere reconfigurar la ciudad desde el barrio, para corregir el rumbo de lo local y, para ello, la discusión en torno al espacio público es obligada, de tal manera que reconquistar el espacio público, como afirma Alguacil (2008), es recobrar la ciudad.

El espacio público urbano se define como todo espacio abierto de valor público, incluyendo recursos hídricos, que ofrece oportunidades de deporte y recreación a las personas y que sirve para el deleite visual de ellas (Alexander, 1977). La calidad de vida en la ciudad depende en gran medida, según Nochian, Mohd, Maulan y Rakhshandehroo (2015), de los espacios abiertos que esta tenga.

El espacio público de los barrios se presenta como lugar de interacción, encuentro, socialización e intercambio entre extraños (Jacobs, 1961; Sampson \& Raudenbush, 
2004; Peters, Elands \& Buijs, 2010). Es el lugar de la comunidad donde se pueden profesar la solidaridad y el diálogo, como también la lucha de distintos intereses y el conflicto por el uso (Lefebvre, 1974; Mitchel, 2003; Oviedo, Rodríguez \& Rodríguez, 2008).

Existen varios conceptos ligados al espacio público: el que se expresa en términos conectivos (sistema de caminos para acceder a la propiedad privada) que se asemeja a un lugar inerte al cual le acontecen cosas y eventos y puede estar despojado de cualidades sociales o estéticas, o en términos de a quién pertenece, si al Estado o a un particular (López de Lucio, 2000). Sin embargo, el espacio público se debería definir de acuerdo con su uso y no solo en términos de titularización de la propiedad o accesibilidad a estos (Carmona, Tiesdell, Heath \& Oc, 2010; Hernández-García, 2013).

$\mathrm{Si}$ monitoreamos nuestras rutinas diarias encontramos ciertas características fundamentales, que incluyen no solo la forma en la que usamos y nos movilizamos en los espacios públicos de la ciudad, sino también -de acuerdo con las circunstancias individuales y la comprensión que tengamos del lugar- las diversas maneras de sentir y de comportarnos en ellos, desde estar a gusto hasta angustiados y caminar con cautela al pasar o permanecer en un determinado lugar (Madanipour, 2003). De ahí que la actitud frente a la seguridad que este produce sea uno de los factores que más influye en el uso del espacio (Jorgensen, Ellis \& Ruddell, 2012).

Hoy en día existe suficiente evidencia para afirmar que el uso del espacio público está correlacionado con la actitud frente a la seguridad, puesto que un espacio concurrido y vital va a hacer sentir a las personas más seguras (Jacobs, 1961; Project for Public Spaces, 2000; Rau, 2005; Gehl \& Svarre, 2013). Teniendo en cuenta este postulado, se hace evidente la necesidad de buscar técnicas y herramientas que permitan medir el uso del espacio público, como estrategia para mejorar la actitud frente a la seguridad de este.

El objetivo de este artículo es hacer una revisión de la literatura en cuatro temas:

1. La noción de barrio y el miedo como operador de la seguridad: se problematizan los espacios públicos del barrio como lugares del miedo.

2. La seguridad como agente de decisión para el uso del espacio: se reflexiona en torno a la correlación entre el uso del espacio y la actitud frente a la seguridad, y se explica que el diseño arquitectónico puede inhibir o promover el uso del lugar.

3. Los mecanismos para medir espacio seguro: se identifican las diversas maneras de medir los espacios públicos y los indicadores de mayor frecuencia de utilización.

4. Las prácticas espaciales cotidianas, también denominadas patrones de conducta habitual, o análisis sistemático de las dinámicas y usos del espacio, que sirven para potencializar su uso.

Las preguntas que de estos temas emergían se pusieron en diálogo con un trabajo empírico de Cárdenas (2016) denominado Ciudad Vital -llevado a cabo en Palmira en 
2014 y en Cali en 2016- para contribuir a la reflexión ampliada acerca del espacio público seguro. Este estudio buscaba medir la correlación entre la actitud de las personas frente a la seguridad y el uso de los espacios públicos urbanos en barrios de alta criminalidad y delincuencia de estas dos ciudades, desde los modelos vitales de ciudad propuestos por la arquitectura, como salida sostenible al diseño de espacios públicos urbanos seguros.

\section{La noción de barrio y el miedo como operador de la seguridad}

Sobrepasadas las esferas individuales del cuerpo, afirma Bailly (1978), el caparazón formado por la habitación y el refugio (casa o apartamento), frecuentado por los seres familiares, el barrio es la primera esfera conformada por un terreno conocido pero no sometido a lo individual. A diferencia de escalas de ciudad más grandes, donde prima la incertidumbre que genera lo desconocido, el barrio es un espacio protector, en el cual las personas se sienten cómodas porque su carácter "familiar" hace que el individuo reconozca a los demás y sea reconocido.

El nivel de proximidad física y de reconocimiento entre las personas del barrio contribuye a que se sientan parte de una comunidad. El tiempo de permanencia en el barrio genera una imagen más concreta y favorable de este. De la misma manera, el desconocimiento del espacio hace que se establezcan dos imaginarios del lugar: el de la "realidad vivida por los residentes del barrio y el de la realidad construida a partir de los flujos inmateriales y los relatos que hablan de él" (Pyszczek, 2012, p. 49). De esta manera, a mayor lejanía física y menor contacto de los ciudadanos con el barrio, más estereotipados y negativos serán los imaginarios acerca de este, porque lo que se desconoce se imagina, se siente y se percibe peligroso.

La arquitectura del miedo y la intimidación (Mitchel, 2003), en gran parte agenciada por el miedo hacia el que se estima diferente, se materializa en formas fortificadas de ciudad, enclaves "entre muros" que conforman barrios cerrados que buscan resguardarse del otro (Caldeira, 2007; Virilio, 2012). Apoyados por las políticas de higienización y bajo la bandera de calidad, homogeneizan los espacios dejando poca cabida para la alteridad, marginalizando y estigmatizando al que no es igual (Bauman, 2006).

"Los miedos no solo son un modo de hablar del mundo sino también una forma de estar en él, de vivir en la ciudad y de relacionarse con las demás personas" (Segura, 2009, p. 66). El miedo al crimen, por ejemplo, es con frecuencia un problema más grave que el crimen propiamente dicho, porque las personas con miedo a ser victimizadas cambian sus hábitos y se inhiben de usar el espacio (Bannister \& Fyfe, 2000). Por ello, el uso del lugar depende en gran medida de la actitud, las percepciones, las representaciones y los imaginarios que se hayan construido sobre este, los cuales no se definen únicamente en términos estadísticos (Aguilar, 2008).

El espacio público en el barrio queda desplazado no solo por el miedo al contacto con el otro, sino por el miedo a usar la ciudad (Harvey, 2013). Más aún, con los dispositivos tecnológicos que virtualizan la experiencia sensorial, el paseo y la conversación 
propias del espacio público, quedan reemplazados por una "soledad compartida por millones de habitantes" (López de Lucio, 2000, p. 2).

La segregación espacial de algunos barrios, la privatización y la especialización del espacio público en ellos -como la llegada del automóvil-, por nombrar algunos factores que complejizan la vida en la ciudad, han ocasionado que la vitalidad de los espacios públicos se reduzca, puesto que su existencia no se puede separar de las prácticas sociales colectivas que en ellos ocurren. Los espacios públicos se usan menos, lo cual es paradójico si se estima que la calidad de vida de una ciudad está estrechamente relacionada con la provisión de espacios verdes que esta brinde. Claro está que se habla de espacios efectivos, no en cantidad, sino en uso.

La falta de uso real de los espacios es resultado, en gran parte, del miedo a interactuar con el desconocido, pero también de la disminución peatonal, la proliferación de espacios monofuncionales, la multiplicación del espacio verde -como un retazo de la retícula de ciudad y su disminución como espacios simbólicos- y la velocidad vehicular, factores que impiden que ocurra el intercambio entre personas (GarcíaJerez \& Peralta, 2014). Esta realidad ha cercenado tanto la existencia como el significado del espacio público en los barrios.

Si como afirma Alguacil (2008) una ciudad segura es aquella donde los espacios públicos son reocupados, transitados y compartidos, entonces la vitalidad de dichos espacios está correlacionada con la actitud frente a la seguridad.

\section{La seguridad como agente de decisión para el uso del espacio}

La literatura indica que existen elementos del ambiente construido que modifican la manera en que nos sentimos en el espacio (Jorgensen et al., 2012). El uso del espacio depende de la actitud que se tenga frente a estos elementos o claves, que pueden ser sociales, como el estereotipo de una persona que coincida con la fisonomía de un ladrón (Rengert, 1980), o ambientales, que tienen que ver con la apariencia física del lugar que incluye edificios abandonados, grafitis, vegetación mal mantenida y basura (Fisher \& Nasar, 1992). Estos signos causan desconfianza frente a la seguridad del lugar y, como consecuencia, se disminuye el uso (Tulloch, 2000). A la arquitectura le interesa estudiar las claves ambientales, porque son las que impactan directamente el diseño arquitectónico.

De acuerdo con el Reporte global de los asentamientos humanos (UN-Habitat, 2007), entre 1980 y 2000 , la delincuencia en el mundo registró un incremento de $30 \%$, y se asegura que $15 \%$ de estas ofensas están relacionadas con el lugar de ocurrencia del delito. De esta manera, se puede afirmar que conforme a las teorías de la oportunidad (Felson \& Clarke, 1998) la ofensa se produce cuando se encuentra la oportunidad contextual. De ahí que sea importante analizar dónde se comete el delito para aumentar el uso de estos lugares y así lograr que el espacio dé la sensación de ser más seguro.

Naciones Unidas (UN-Habitat, 2015b) reconoce la correlación entre la seguridad y el diseño del ambiente construido, cuando afirma que se puede reducir la delincuencia y 
la violencia si los espacios públicos urbanos están bien diseñados y mantenidos, mientras que cuando no hay suficiente espacio público en la ciudad, este ha sido privatizado o está mal diseñado, la ciudad se segrega, se dejan de usar los espacios, y la violencia y la delincuencia aumentan.

Conforme a lo que plantea Naciones Unidas, en Colombia existe la Política Nacional de Seguridad y Convivencia Ciudadana (Departamento Nacional de Planeación, 2012) que reconoce la provisión y la renovación de los espacios públicos en los barrios como una estrategia de prevención del delito, porque la seguridad es garante del uso del espacio, puesto que un lugar no se frecuentaría a voluntad si se estima peligroso (Jorgensen, Ellis \& Ruddell, 2012). Esta política busca la prevención situacional del delito en los espacios públicos urbanos, es decir, no pretende conocer las razones por las que una persona comete el delito, sino que atiende los contextos (el lugar físico) donde estos ocurren.

Gehl, Gemzoe, Kirknaes y Sondergaard (2006), por su parte, proponen doce criterios para mejorar la calidad de los diseños de espacio público. Los agrupa en tres dimensiones: la protección, el confort y el placer. En la primera dimensión, denominada protección del crimen y la violencia - sensación de seguridad, nuevamente se establece una correlación entre la vitalidad entendida como el uso del espacio y la actitud frente a la seguridad.

Sin embargo, la pregunta que subyace es ¿cómo medimos los espacios públicos urbanos seguros?

\section{Mecanismos de medición de los espacios públicos}

Para avanzar hacia el diseño de espacios públicos urbanos seguros, se hace imprescindible que los arquitectos y los gobiernos evalúen la cantidad y la calidad del espacio público (UN-Habitat, 2015a). Para ello, hay que ponerse de acuerdo en la elaboración de herramientas e indicadores y, en este sentido, el mundo académico y la investigación desempeñan un papel clave.

Medir el espacio con relación a la seguridad no es novedad. En la década de 1970 se estudió ampliamente cómo el ambiente construido influenciaba el comportamiento de las personas. Al respecto, Jeffrey (1977) estableció pautas tipológicas para prevenir el crimen a través del diseño, y Newman (1975; 1995; Newman y Franck, 1982), a su vez, lo hizo estudiando el comportamiento de los residentes en edificios subsidiados por el Estado, en respuesta al alarmante incremento en los índices de criminalidad en los Estados Unidos. En su libro Espacio defendible (Newman, 1973) se advierte que la probabilidad de crimen aumenta cuando los edificios superan los cinco pisos y se encuentran rodeados de grandes extensiones de terreno. Sin embargo, estas propuestas metodológicas están más encaminadas a viviendas y a su relación con el entorno inmediato, no a espacios públicos urbanos.

En cuanto al espacio público urbano, Villanueva et al. (2015) indican que tradicionalmente existen cinco maneras de medirlo: (a) la distribución de los espacios públicos urbanos en la ciudad, donde lo que se busca es determinar si estos están 
equitativamente distribuidos; (b) el porcentaje de área reservada a espacio público sobre el total de suelo urbano de la ciudad; en Colombia, esta distribución se encuentra contenida en los planes de ordenamiento territorial, en los que se estima un porcentaje obligatorio de cesión de tierra a espacio público, determinado por el índice de construcción de las edificaciones; (c) los radios poblacionales que miden área verde con relación a la cantidad de población; (d) la distancia máxima que pueden tener las residencias al espacio público más cercano; esta es la más utilizada en los países del norte y en algunas ciudades de Australia; y (e) la cantidad de mobiliario urbano de cada lugar; en esta medida se estima que a mayor número de canchas, juegos, bancas y espacios comunitarios, mejor es la calidad del espacio público urbano. En Colombia se cuenta con indicadores que miden el espacio público, como el de compacidad, que establece la relación entre volumen edificado y área de espacio efectivo (Alcaldía Mayor de Bogotá D.C., 2016).

Bajo estas lógicas se han realizado esfuerzos de mapeo de los espacios, utilizando el GIS, software de ESRI (Environmental System Resource Institute), con el que actualmente cuentan las ciudades colombianas de Cali, Medellín y Bogotá, entre otras.

Otro importante aporte a los indicadores urbanos en la región lo realiza el Banco Interamericano de Desarrollo (2016), con la Iniciativa Ciudades Emergentes y Sostenibles (ICES), que es una estrategia para la asistencia y gestión encaminada al crecimiento sostenible de las ciudades emergentes de América Latina y el Caribe. La estrategia cuenta con una metodología en la que se emplean indicadores en tres áreas: sostenibilidad ambiental y cambio climático, sostenibilidad urbana, y sostenibilidad fiscal y gobernabilidad. Estos indicadores tienen el propósito de identificar aspectos que constituyen los mayores desafíos para la sostenibilidad de una ciudad.

Entre los indicadores que le corresponden a la dimensión de sostenibilidad urbana se encuentran los de uso del suelo, los cuales miden la expansión de la huella urbana, definida como el crecimiento del área oficial urbana dentro de la ciudad. Este indicador está orientado hacia una mejor planificación urbana. La ICES también utiliza indicadores que miden el porcentaje de áreas verdes y la cantidad de espacios públicos por cada 100.000 habitantes; por lo tanto, apuntan a una distribución más equitativa de estos. Si bien estos indicadores son de suma relevancia en términos de planificación urbana para lograr una correcta y equitativa dotación de espacios verdes en la ciudad, difícilmente se podría inferir de ellos el porcentaje de uso de estos espacios; en tal sentido, más espacio verde no significa necesariamente mayor uso de estos.

Más aún, los indicadores de seguridad son comunes, la ICES, por ejemplo, mide tasas de homicidio, violencia intrafamiliar y violencia física contra mujeres, robos, hurtos y percepción de seguridad -definida como el porcentaje de ciudadanos que se sienten seguros y que se obtiene a través de encuestas-. Sin embargo, no se encontraron indicadores que midieran el uso con relación a la seguridad, es decir, que determinaran cómo el uso del espacio modifica la actitud frente a la seguridad, y es en este sentido que va el aporte de la investigación de Ciudad Vital, la cual propuso un modelo de 
medición de variables de vitalidad asociadas a la seguridad tanto para Palmira como para Cali.

Volviendo a los indicadores de uso, entre las aproximaciones cualitativas se encuentran otros autores como Wood, Frank y Giles-Corti (2010), quienes afirman que existen cualidades de unos espacios que los hacen más atractivos que otros, y que esto contribuye a que se usen más. Porta y Renne (2005), por su parte, desarrollaron los indicadores formales para medir las representaciones gráficas de los lugares. Estos indicadores separan los atributos del diseño para identificar los factores positivos y negativos presentes en el lugar. Sin embargo, se advierte que, al ser una herramienta, su función no es la de valorar los diseños en buenos o malos, sino aportar a la discusión local, para definir cuáles son los atributos positivos que pueden servir a un determinado contexto de ciudad.

El índice formal de Porta y Renne (2005) está compuesto por dos indicadores. El primero, denominado indicador de tejido urbano, estudia los factores de tamaño, accesibilidad, permeabilidad y conectividad vial (Hillier \& Hanson, 1984; Hillier, 2004), el uso mixto (Gehl \& Svarre, 2013), la proporción de espacio público frente al espacio privado, el número de edificios y de espacios vacíos del sector, y la vigilancia natural u "ojos en la calle", término acuñado por Jacobs (1961) para referirse al control social informal que las personas puedan ejercer sobre el territorio, bajo la hipótesis de que los espacios concurridos y vitales se sienten más seguros (Sampson \& Raudenbush, 2004).

El otro indicador es el de las calles, el cual mide la cantidad de cielo visible desde la calle que hace sentir a las personas protegidas y a gusto; la continuidad de las fachadas, la transparencia y el espacio de transición (Carmona et al., 2010), que es el ancho social o espacio entre la fachada y la calle, donde se puede dar la actividad social; la complejidad visual, que incluye color, tipos de fachada y de pavimento. Por último, la medida de sedibility, término en inglés utilizado por Whyte (1980) para hablar de la posibilidad que ofrece el lugar para sentarse, puesto que para grupos como el The Project for Public Spaces (2000), el principio de vitalidad indica que nos sentamos donde otros se sientan y nos gusta estar donde otros están.

Estos acercamientos incluyen los recorridos sobre planos, utilización de dibujos y fotografías para la construcción de mapas mentales que constituyen un indicador de las representaciones socioespaciales del lugar (De Alba, 2004). Sin embargo, estos mapas están más interesados en describir y comprender las relaciones cambiantes de las personas en el lugar, que en medir el uso del espacio.

Entre las herramientas más utilizadas por la arquitectura para medir cualitativamente el espacio predominan los diseños participativos, que consisten en apoyarse en la construcción de cartografías sociales, mediante entrevistas, mapeos y otros para diseñar conjuntamente el lugar. Esta metodología ha probado ser muy útil para las etapas de diagnóstico de las intervenciones de renovación de barrios, pero no garantiza el uso del espacio; de hecho, pareciera que persiste una brecha entre el pedido de la comunidad y las formas arquitectónicas resultantes, lo que indica que para que un diseño sea exitoso debe incorporar mecanismos para que sea apropiado 
por los residentes. Por eso, grupos como The Project for Public Spaces (2000) y Gehl (2011; 2013) Architects, reconocidos por sus numerosas y exitosas renovaciones urbanas alrededor del mundo, advierten que un indicador de apropiación de los diseños es el aumento en uso del espacio, y que este difícilmente se consigue mediante el informe verbal proveniente de la participación, sino a través de la atenta observación de las conductas y movimientos en el lugar.

Los grupos anteriormente mencionados reconocen que el espacio público urbano está marcado por inercias, dinámicas y fronteras espaciales (Oviedo et al., 2008), de tal manera que no es un lugar inerte al que le acontecen cosas y eventos, sino que es un lugar que se modifica constantemente, con dinámicas propias y capaz de dar cuenta de las asimetrías de poder que se producen en su uso (Carrión, 2012). Lo que afirman Gehl (2013) y The Project for Public Spaces (2000) es que los diseños que reconocen estas prácticas espaciales cotidianas tienen mayor posibilidad de éxito en términos de uso, puesto que estudian la manera en que las personas viven y se mueven naturalmente en el espacio, lo que posiblemente hará que estos residentes no sientan extraño el diseño a su diario vivir.

\section{Prácticas espaciales cotidianas o patrones habituales de conducta}

Berneth (2016) sostiene que los usos del espacio dependen, entre otros, de lo que él denomina intencionalidad securitaria, que son "acciones deliberadas que tienen como objetivo contrarrestar la inseguridad personal a través de la vigilancia" (p. 105). Esta intencionalidad está compuesta por tres dimensiones: (a) la forma de presentarse o actuar en el lugar-también denominadas en este artículo claves sociales-, (b) el uso de expresiones verbales, y (c) las prácticas espaciales cotidianas. Las primeras dos sirven para medir percepción de seguridad en el espacio, mientras que la tercera dimensión mide las apropiaciones espacio-temporales que las personas hacen de la ciudad, en otras palabras, la manera en que usamos el espacio.

El estudio de las prácticas espaciales cotidianas permite observar las dinámicas y los usos del lugar para establecer un patrón habitual de conducta (Carnap, 1966), el cual indica qué tan vital es el lugar (Whyte, 1980; Project for Public Spaces, 2000; Gehl \& Svarre, 2013).

Para medir el patrón habitual de conducta se utilizan técnicas de observación. Al respecto, Anguera (1978) sostiene que estas "aportan datos que atañen directamente a situaciones de comportamiento típico" (p. 25). Esto significa que las conductas que se observan responden a un cierto patrón que es predecible, así las conductas en ambientes similares admitan un grado de generalización, o lo que Carnap (1966) llama regularidad casual, la cual reconoce, mediante la observación sistemática, cuáles son las dinámicas cotidianas del espacio, cómo se mueven las personas, dónde se sientan, qué espacios usan, cuáles son las horas de mayor actividad y a qué grupos poblacionales se está atendiendo principalmente, que se puedan traducir a un diseño que potencialice el uso del espacio. Para ello, la mayoría de las investigaciones que estudian el uso con relación a la actitud frente a la seguridad (Fisher y Nasar, 1992; Project for Public Spaces, 2000; Gehl y Svarre, 2013) sugieren establecer el patrón 
habitual de conducta o, como lo denomina Berneth (2016), las prácticas espaciales cotidianas. Al respecto, García-Jerez (2016) estima que:

El habitus ambulante estará conformado por las prácticas cotidianas de movilidad, es decir, las rutas seleccionadas, los dispositivos de desplazamientos utilizados, la interacción que establecen los individuos con esos mismos dispositivos y con otros individuos; pero también formarán parte de este habitus, tal y como ya se enunció, las representaciones que poseen los sujetos acerca de esos recorridos, de la propia noción de movilidad [...] (p. 30).

Si bien este autor se está refiriendo a la movilidad vehicular, este mismo principio aplica para la movilidad peatonal, en cuanto a que el patrón habitual de conducta registra ese habitus ambulante conformado por las prácticas cotidianas de movilidad de las personas. De hecho, podría indicarse que no existe la distinción entre movilidad peatonal y la motorizada, tal y como ya lo anunció Erving Goffman (1979) cuando afirmaba que "una unidad vehicular es un caparazón de algún tipo controlado (por lo general desde dentro) por un piloto o un navegante humano" (Goffman, 1979, p. 26).

Para medir el patrón habitual, los métodos observacionales no intrusivos son útiles, porque sirven para registrar conductas que se modificarían si el sujeto de la investigación conociera que está siendo observado (Webb, Campbell, Schwartz \& Sechrest, 1996). Estas medidas son una manera de conocer el patrón habitual de conducta, sin tener que recurrir al testimonio, ya que este podría estar viciado por intereses particulares o ser impreciso porque a veces lo que se dice no coincide con lo que se hace.

La observación no intrusiva es especialmente útil cuando se está investigando un tema sensible para la comunidad, como la seguridad, en el que existe la posibilidad de resistencia por parte de personas o grupos. Este tipo de observación se puede llevar a cabo mediante el empleo de cámaras localizadas estratégicamente, como en el caso de Whyte (1980), que las empleó para conocer el comportamiento de las personas en los espacios urbanos. Este autor utilizaba el "time-lapse", técnica en la que se programa una cámara para tomar fotos en intervalos de tiempo preestablecidos (por ejemplo, cada 10 minutos en un periodo de 48 horas seguidas). La observación también se puede realizar a través de visitas de campo (Project for Public Spaces, 2000; Gehl, 2011; 2013), como se hizo en la investigación de Ciudad Vital.

\section{Prácticas espaciales cotidianas en Ciudad Vital: los casos de Palmira y Cali}

En Palmira (2014) y en Cali (2016) se realizaron investigaciones que buscaban medir la correlación entre la actitud de las personas frente a la seguridad y el uso de los espacios públicos urbanos en barrios de alta criminalidad y delincuencia. Estas investigaciones tenían como propósitos establecer premisas proyectuales para el diseño de espacios públicos urbanos seguros sin perder la vitalidad y proponer estrategias a los gobiernos municipales para la evaluación de estos diseños. 
Para medir la actitud frente a la seguridad se utilizó la medición de actitudes del diferencial semántico (Osgood, Tannenbaum \& Suci, 1957), y para medir el uso de los espacios se recurrió a la observación sistemática no experimental (Anguera, 1978), de manera no intrusiva (Webb et al., 1996), con el fin de establecer las prácticas espaciales cotidianas, que en Ciudad Vital se denominan patrones habituales de conducta.

Los resultados de esta investigación indicaron que existen seis variables del ambiente construido que afectan la actitud frente a la seguridad en estas dos ciudades (Cárdenas, 2016). Para la ciudad de Palmira se identificaron las siguientes variables: (a) densidad de personas por área $(r=0,82)$; (b) provisión de espacios de transición ( $r=0,82$ ); (c) tipología en culs de sacs (vías de un solo tramo que potencializan la interacción entre residentes); (d) limpieza y mantenimiento $(r=0,96)$; (e) visibilidad en los bordes $(r=0,90)$; y (f) cantidad de patrullas en el área $(r=-0,80)$, esta correlación negativa indica que a mayor número de patrullas en el lugar peor es la actitud frente a la seguridad; y la baja correlación entre el encerramiento y la seguridad $(r=0,26)$, lo que mostró que el número de rejas no genera una mejor actitud. Las primeras cinco variables fueron las claves ambientales de uso que más afectaron la actitud frente al espacio público en términos de seguridad. La sexta variable tenía relación con las barreras físicas.

Los resultados en Cali fueron muy similares. Las correlaciones alcanzadas sugirieron que las formas arquitectónicas que promueven mayor vigilancia natural, entendida como la voluntad de ayudar a alguien conocido o no, de requerirse, generan mejor actitud frente a la seguridad.

Para establecer el patrón habitual de conducta en Ciudad Vital fue necesario efectuar mediciones en diferentes días de la semana y en diferentes horarios, de ahí su diseño longitudinal. Se efectuaron medidas durante la semana, en fin de semana y en feriados, porque el comportamiento y el tránsito habitual de personas dependen del día de la semana. También fue importante hacer la medición en horarios siempre iguales, como los diez primeros minutos de cada hora, y registrar las condiciones climáticas durante la toma, puesto que pueden influir en el uso.

Para la observación sistemática del patrón habitual de conducta se utilizó una serie de protocolos de observación que fueron tomados de investigaciones similares en el mundo (Whyte, 1980; Fisher \& Nasar, 1992; Project for Public Spaces, 2000; Gehl, 2011; Gehl \& Svarre, 2013), pero contextualizados mediante un piloto a la realidad de Palmira y Cali.

Para medir la densidad de personas por área se utilizó una ficha de recolección de datos que indica la cantidad de personas en el espacio y la actividad que realizan (tabla 1).

Esta información se desagrega en género, edad y características de la actividad, las cuales se dividen en: (a) necesarias, que son las que exigen algún tipo de obligatoriedad (como tomar el bus para ir al trabajo), y (b) no necesaria o a voluntad (como leer un periódico). Los espacios con mayor número de personas realizando 
actividad no necesaria se estiman más seguros, puesto que, como ya se advirtió, no se frecuentaría un espacio a voluntad si se percibe inseguro. Esta ficha también informa si la actividad es socialmente aceptada o no, ya que a mayor presencia de actividad socialmente no aceptada en un lugar, peor es la actitud frente a la seguridad. En términos de diseño arquitectónico, mediante el estudio de esta ficha se puede conocer con más claridad quién y cómo cotidianamente se usa el espacio, para hacer diseños más ajustados al tipo de usuario y a la actividad del lugar.

Tabla 1. Ficha de recolección de datos

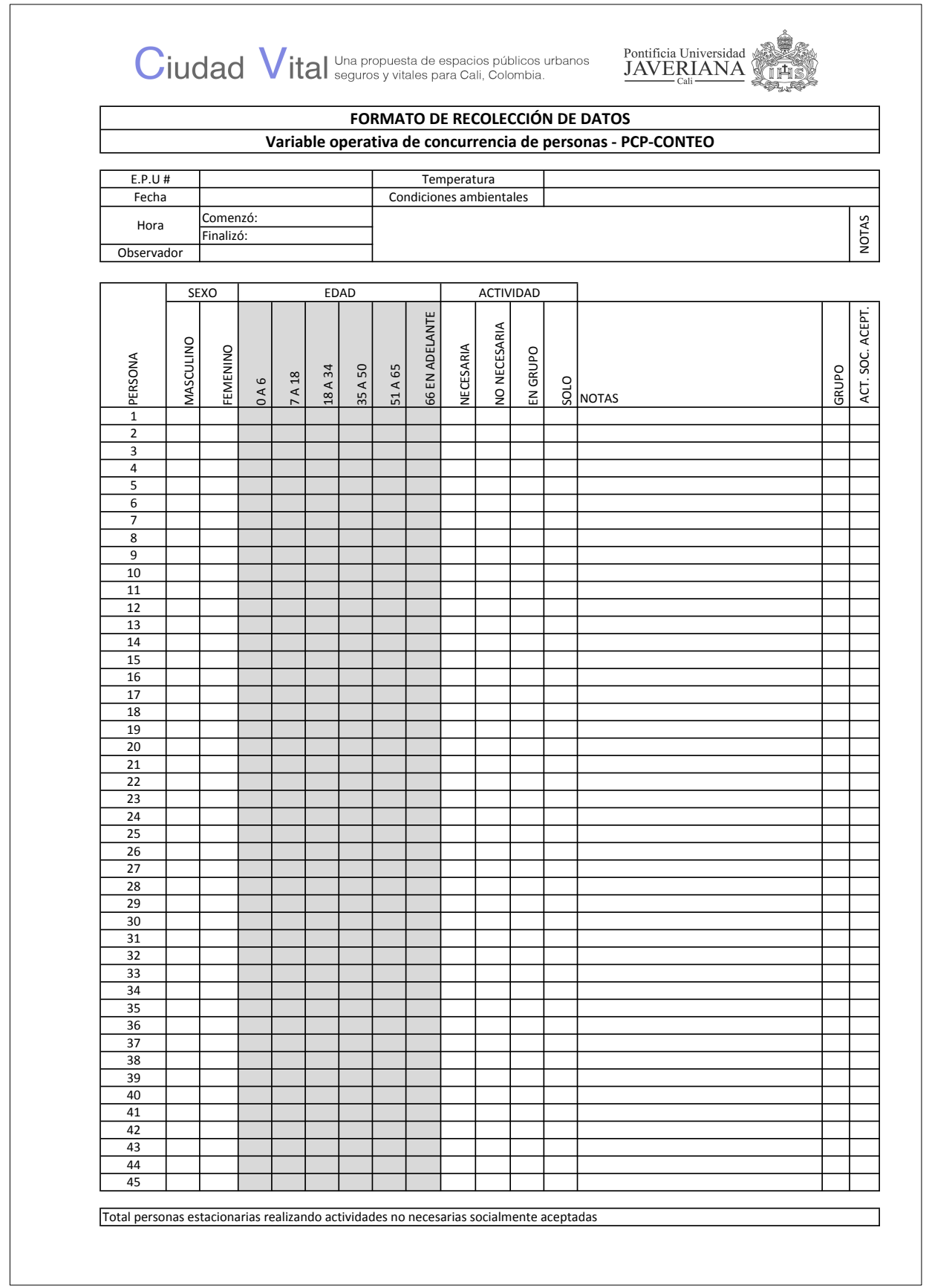

Fuente: elaboración propia, adaptación del protocolo de Project for Public Spaces (2000), para la investigación de Ciudad Vital. 
La información obtenida con la ficha de recolección de datos fue corroborada con los planos de trazabilidad (figura 1), que indican cuáles rutas toman las personas y cuáles esquivan, es decir, sus rutas de deseo. El análisis en conjunto de la ficha de recolección de datos y de los planos de trazabilidad arroja luz acerca de las posibles razones para las decisiones en cuanto a la movilidad en el lugar. Estas decisiones se deben a razones que pueden ser sencillas -como la presencia de bancas o vegetación que obstaculicen la ruta habitual y que deban ser reorganizadas en el nuevo diseñoo más complejas -como la presencia de posibles barreras simbólicas de los territorios, que son las líneas invisibles que marcan las pandillas y por donde solo pueden transitar los que pertenecen a ellas, en tal caso, el diseño deberá contemplar este fraccionamiento del lugar-.

Figura 1. Planos de trazabilidad de líneas del deseo

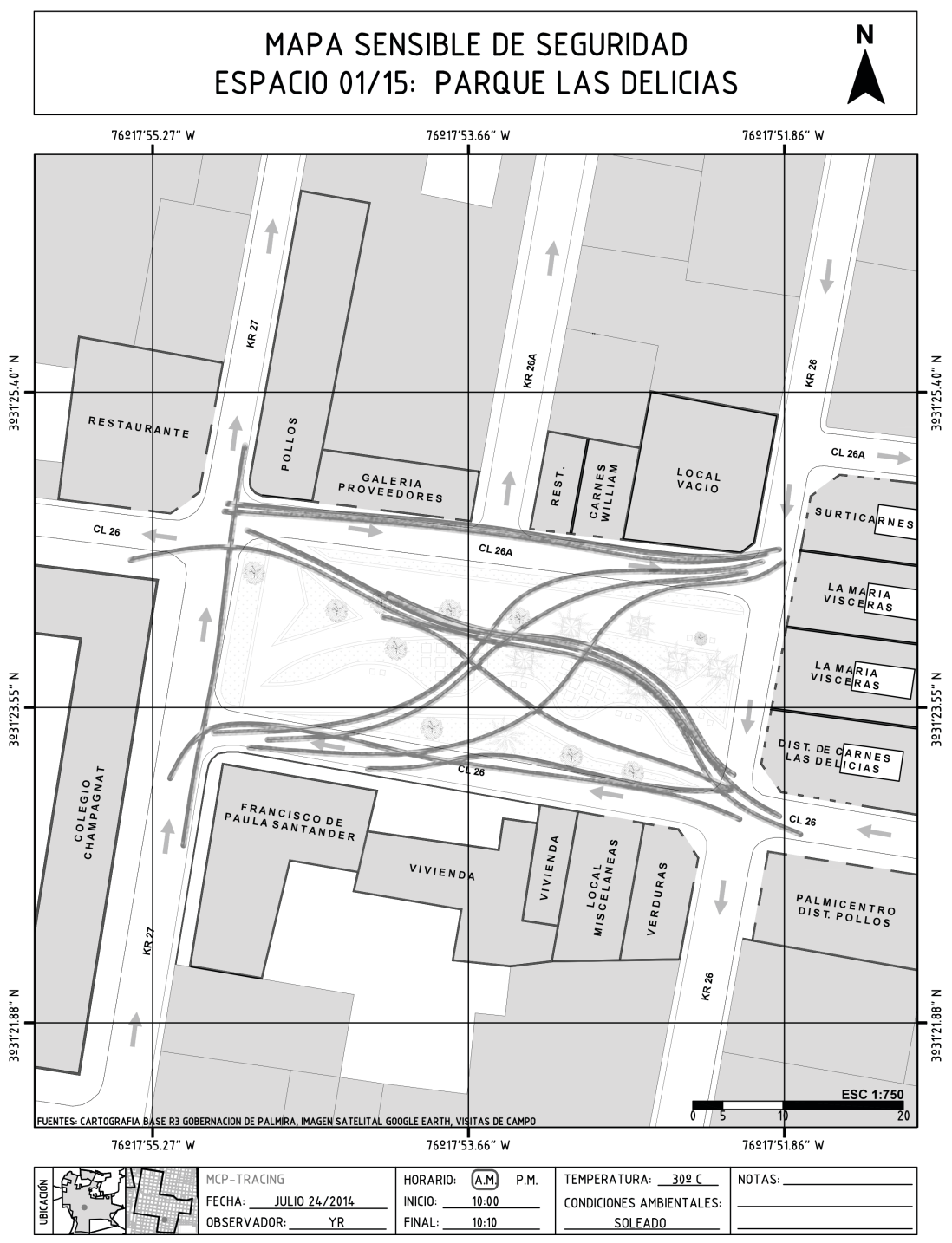

Fuente: elaboración propia, adaptación de los planos de trazabilidad de Gehl y Svarre (2013), para la investigación de Ciudad Vital. 
Los planos de usos sirven para medir la provisión de espacios de transición, es decir, los antejardines, porches o tiendas en el primer piso, donde se produce mayor interacción social, que haría sentir el espacio más concurrido y a su vez más seguro. Estos planos se cotejan con los de aglomeración y dispersión (figura 2), en los cuales se marca toda persona sentada en el lugar durante el tiempo que dure la toma, desagregando la información en términos de género. Este plano indica los sitios donde las personas prefieren estar; decisión que puede obedecer a la presencia de un árbol que genere sombra, aleros de las edificaciones o espacios abiertos que promuevan una cierta actividad. El estudio de estos planos ayuda a detectar la correcta localización del mobiliario urbano, equipamientos y escenarios deportivos que deberían corresponder con estos lugares de mayor aglomeración.

Figura 2. Plano de patrones de aglomeración y dispersión

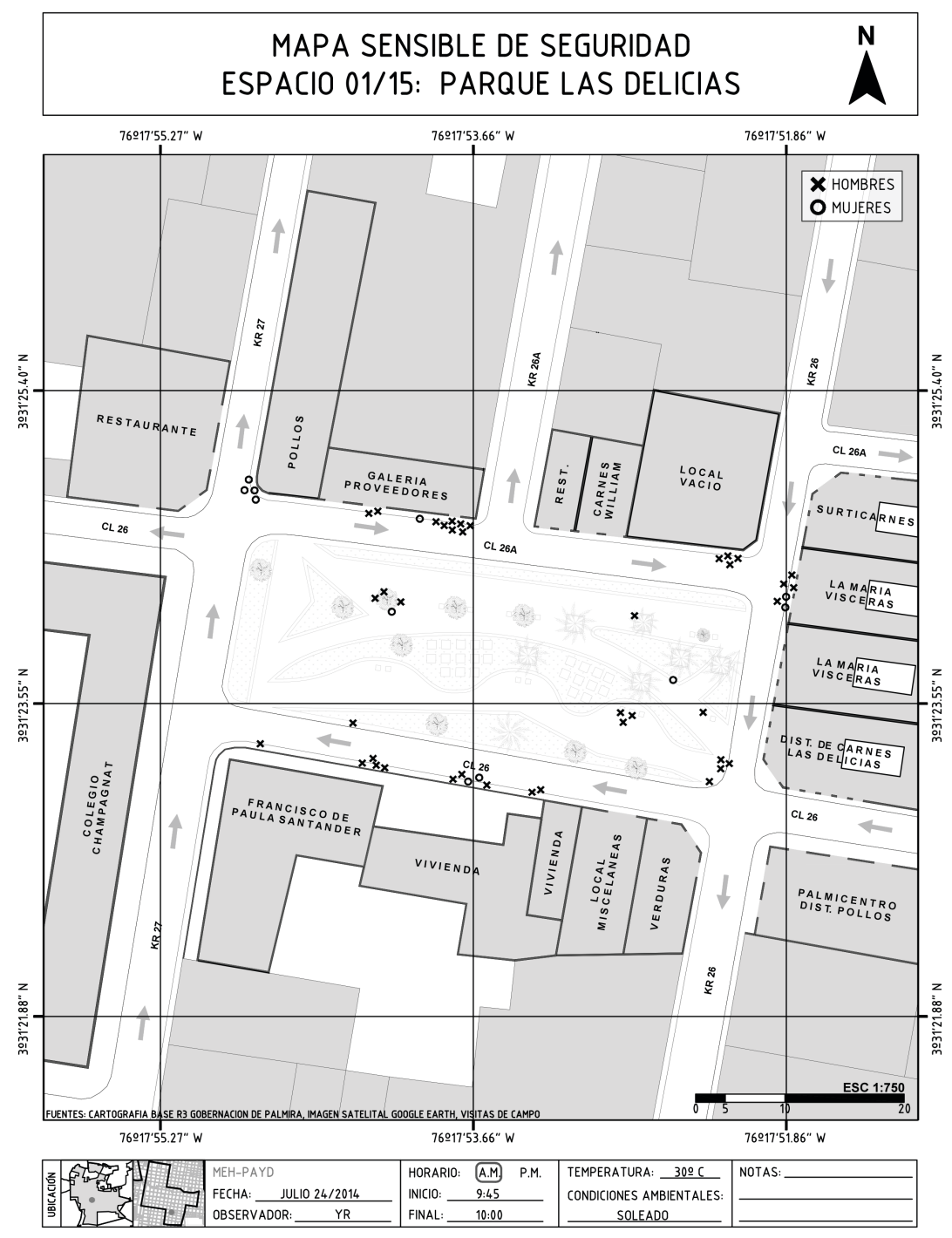

Fuente: elaboración propia, adaptación de las herramientas de Gehl y Svarre (2013), para la investigación de Ciudad Vital. 
Para medir la tipología de vías se trazó el recorrido vial sobre el plano general de los espacios de estudio y se marcó el número de segmentos de vías presentes en el lugar. En ambas investigaciones, a mayor cantidad de segmentos peor es la actitud frente a la seguridad. Este plano sirve para reducir la cantidad de espacio en movimiento, que es el producido por el coche, y para promover el espacio social mediante la peatonalización de vías y conversión de estas a tránsito lento o restringido, sobre todo en zonas residenciales.

Para medir la iluminación se construyó un catálogo de postes de luz presentes en los espacios estudiados, con el que se midió el radio de luz que generaba cada luminaria. Así se pudo conocer tanto el porcentaje de espacio iluminado del lugar como el que se encontraba a oscuras. Con este plano se puede identificar dónde se requiere luz adicional. La investigación de Ciudad Vital demostró que a mayor iluminación de los andenes, senderos y zonas de transición, principalmente, mejor es la actitud frente a la seguridad. Este plano analizado en conjunto con los planos de patrones de aglomeración y dispersión y con los planos de trazabilidad permite ubicar, redistribuir y relocalizar las luminarias para potenciar el uso del lugar.

La limpieza y el mantenimiento se midieron a través del diferencial semántico con el que se obtuvo una lista descendente, de mejor a peor, de los espacios que generaban mejor sensación de limpieza y se consideraban bien mantenidos. Luego se organizaron por orden de registro para conocer qué era lo que más impactaba la actitud frente a la limpieza y el mantenimiento (basura, grafitis, edificios abandonados, vegetación mal mantenida). En los casos de Palmira y Cali, la falta de corte de césped fue lo que más impactó la actitud, seguido de la presencia de basura. Conocer esta información permite realizar un diseño paisajístico de bajo mantenimiento y disponer correctamente los cestos de basura de acuerdo con el plano de aglomeración y dispersión.

\section{Reflexiones}

Conocer las características del lugar permite tomar decisiones más precisas con respecto a la localización de los elementos que conforman un diseño arquitectónico. Este es el tipo de información al que difícilmente se puede acceder mediante el informe verbal y por eso es tan importante la observación como herramienta de diseño.

La observación sistemática hace posible identificar cómo les gusta a las personas usar el lugar y potencializa aquellos espacios que tienen una vida propia. Medir las dinámicas del lugar permite realizar diseños que respeten e incorporen los usos cotidianos del espacio, como estrategia para que la comunidad los acepte mejor. La observación sistemática derivada de estas herramientas se puede traducir en pautas de diseño para incrementar el uso y, por lo tanto, mejorar la actitud frente a la seguridad en el lugar. Esas pautas incluyen: 
- Aumentar la densidad de personas por área, por ejemplo, dividiendo el espacio en clústeres de diferentes actividades para atraer al lugar la mayor población heterogénea posible (por género, edad y actividad) y que no se monopolice el espacio con una sola actividad; lo cual se hace mediante el estudio del plano de patrones de aglomeración y dispersión.

- Incrementar tanto el número de mobiliario para sentarse cerca de las rutas de tránsito habitual y no obstaculizarlas con mobiliario o juegos, lo cual se hace mediante la revisión atenta del plano de trazabilidad.

- Rediseñar los andenes sobre las líneas de tránsito habitual o líneas del deseo que aparecen en el plano de trazabilidad y que marcan las rutas por donde las personas prefieren caminar.

- Diseñar andenes más amplios o espacios de transición entre los bordes y el espacio público en los sitios identificados en los planos de trazabilidad como de mayor concentración de las líneas del deseo. Por ejemplo, cuando se estaba diseñando la renovación del Times Square en Nueva York, el plano de trazabilidad en los diferentes horarios dio tal intensidad en el flujo peatonal que justificó la peatonalización de varias cuadras.

- Nivelar algunos andenes a la altura de los espacios públicos para generar mayores zonas de transición. Este fue el caso del diseño de la peatonalización del centro de Palmira, seleccionado en la Bienal de Arquitectura como un buen ejemplo de renovación urbana.

- Disponer de luminarias y elementos para el aseo, como cestas de basura, en las rutas de tránsito habitual y en los lugares de mayor aglomeración de personas.

- Realizar diseños paisajísticos de bajo mantenimiento.

En términos de Gobierno, estas herramientas podrían permitir:

- Evaluar los diseños de espacio público seguro de acuerdo con el aumento en la frecuencia de uso del espacio.

- Advertir fronteras invisibles que impidan el paso normal de peatones mediante la lectura de las secuencias de trazabilidad. Identificar estar fronteras le sirve al Gobierno para priorizar lugares de intervención.

- Identificar, con la ayuda de las fichas de patrones de aglomeración y dispersión del espacio y de la ficha de recolección de datos, los grupos que estén usando substancias psicoactivas, e incentivarlos a realizar labores para la comunidad -como el corte del césped- a cambio de dejarlos permanecer en el lugar. Esto sirve para enfrentar el problema sin generar desplazamientos de estas personas a otros lugares de la ciudad.

- Incentivar la generación de usos adicionales. Apoyar y ayudar a identificar posibilidades de uso comercial formal o móvil entre moradores.

Este artículo buscó mostrar los avances en la medición del uso del espacio público a través de la observación del patrón habitual de conducta, con el que se pueden determinar pautas de diseño que incrementen el uso del lugar. 
Las herramientas que aquí se presentan y que fueron utilizadas en la investigación de Ciudad Vital, tienen como objetivo ayudar a generar diseños arquitectónicos y urbanos que incorporen los modos de vivir el espacio de las personas del barrio, con el fin de aumentar el uso y la apropiación de estos. Sin embargo, se advierte que no son exhaustivas, no tienen carácter prescriptivo ni suponen ser la única manera de medir el uso del espacio, más bien son complementarias a los talleres y grupos focales durante las etapas de diagnóstico de los diseños de espacio público.

Dado que el diseño arquitectónico es útil en la medida en que se pueda medir el impacto que este tiene en el bienestar y el desarrollo de una comunidad (Jennings, Larson, \& Yun, 2016), se deben crear herramientas e indicadores para saber si el diseño promueve un desarrollo más sostenible o, por el contrario, la inhibe. El éxito de un diseño se evalúa conforme al incremento en la frecuencia de uso. De ahí la importancia del estudio de los patrones habituales de conducta, porque contribuyen a diseñar espacios con un nivel de apropiación y aceptación más alto por parte de la comunidad.

El buen diseño, entendido como el correcto ensamble entre hábitos de uso y vida del lugar con las formas (Alexander, 1977), es aquel que logra ajustarse mejor a las necesidades y usos cotidianos de las personas en los lugares; por eso es necesario prestar atención a lo que acontece cotidianamente en el lugar. Es en este sentido que un estudio de observación sistemática de las dinámicas y las conductas de las personas, antes de iniciar cualquier ejercicio proyectual, es indispensable si lo que se busca son diseños que realmente estén en consonancia con los hábitos de uso.

La correcta utilización de las herramientas observacionales para establecer el patrón habitual de conducta, como complemento de las aproximaciones participativas iniciales del proyecto, puede ayudar no solo a que el resultado del diseño final se aproxime más a las necesidades de las personas, sino a que sea un verdadero reflejo de las decisiones y opciones que la comunidad tiene frente a sus espacios de esparcimiento y de ciudad. Los diseños que están en consonancia con los usos del lugar generan un círculo virtuoso, donde el mayor uso agenciado por el diseño genera una mejor actitud frente a la seguridad, y viceversa.

Se debe reconocer que la responsabilidad por la seguridad en la ciudad no solo está en las manos de los gobiernos municipales, sino también en las de otros actores, como la comunidad y la academia. De tal manera que todos aportemos para vivir en ciudades más seguras.

La creación de estas medidas representa un intento por solucionar algún problema de índole práctico, bien sea social o físico, que no se funde únicamente en satisfacer un deseo de conocimiento científico, sino que provenga de una necesidad cotidiana real (DeVellis, 2016), que en este caso es el diseño del espacio público seguro de nuestros barrios. Es por esta razón que se hace necesario que cada disciplina desarrolle maneras de medir, herramientas e instrumentos especializados para solucionar problemas que le competen a su disciplina y se espera que este artículo vaya en esa dirección. 
El patrón habitual de conducta permitirá identificar la necesidad de dotar la ciudad de espacios públicos que empoderen al otro, reconociéndole sus lógicas, sus modos de hacer y de usar la ciudad, para que pueda tomar sus propias decisiones de vida. También contribuirá a que los diseños den cuenta de las dinámicas de los habitantes con las que se garantice su uso, como estrategia para recuperar el espacio público a escala local.

\section{Referencias}

Aguilar, F. (2008). Representaciones de la inseguridad y la violencia entre los habitantes del fraccionamiento Residencial Pinos del Norte, Mérida, Yucatán. Revista Polis, 7(20), 19-32.

Alcaldía Mayor de Bogotá D.C. (2016). Observatorio del Espacio Público de Bogotá. Recuperado de observatorio.dadep.gov.co

Alexander, C. (1977). A pattern language: Towns, Buildings, Constructions. New York, US: University Press.

Alguacil, J. (2008). Espacio público y espacio político. La ciudad como el lugar de las estrategias de participación. Revista Polis, 7(22), 199-223.

Anguera, M. T. (1978). Metodología de la observación en las ciencias humanas. Madrid, España: Cátedra.

Bailly, A. S. (1978). La percepción del espacio urbano. Madrid, España: Nuevo Urbanismo.

Banco Interamericano de Desarrollo. (2016). Anexo de indicadores. Guía metodológica ciudades emergentes y sostenibles. Iniciativa de ciudades emergentes y sostenibles. Washington D.C, Estados Unidos: BID.

Bannister, J. \& Fyfe, J. (2000). Fear and the city. Urban Studies, 38(5-6), 807-813.

Bauman, Z. (2006). Liquid Fear. Cambridge, UK: Polity.

Berneth, L. (2016). Securonormatividad: la sociabilidad urbana vista a través de las prácticas de la vigilancia cotidiana. URVIO Revista Latinoamericana de Seguridad Ciudadana, (18), 104-123.

Buraglia, P. (1998). El barrio desde una perspectiva socio espacial. Hacia una redefinición del concepto. Recuperado de http://www.barriotaller.org.co

Caldeira, T. (2007). Ciudad de muros. Barcelona, España: Gedeisa.

Cárdenas, S. (2016). La vitalidad como alternativa a la seguridad de los espacios públicos urbanos: el caso Palmira - Colombia. Prospectiva. Revista de trabajo social e intervención social, (21), 157-179. 
Carmona, M., Tiesdell, S., Heath, T. \& Oc, T. (2010). Public places, urban spaces. Oxford, UK: Elsevier.

Carnap, R. (1966). An introduction to the philosophy of science. New York, US: Basic Books.

Carrión, F. (2012). Barrios seguros para mujeres. URVIO Revista Latinoamericana de Seguridad Ciudadana, (11), 33-42.

De Alba, M. (2004). Mapas mentales de la ciudad de México: una aproximación psicosocial a estudio de las representaciones espaciales. Estudios demográficos y urbanos, 19(55), 115-143.

Departamento Nacional de Planeación. (2012). Documento CONPES 3718 Política Nacional de Espacio Público. Bogotá, Colombia: DNP.

DeVellis, R. (2016). Scale development. Theory and application (4 ed.). North Carolina, US: SAGE .

Felson, M. \& Clarke, R. V. (1998). Opportunity makes the thief: practical theory for crime prevention. Police Research Series, Paper 98. London, UK: Research, Development and Statistics Directorate.

Fisher, B. S. \& Nasar, J. L. (1992). Fear of crime in relation to three exterior site features: Prospect, refuge and escape. Environment and Behavior, 24(1), 35-65.

García-Jerez, F. A. (2016). La movilidad socio espacial desde la teoría de Pierre Bourdieu: capital de motilidad, campo de movilidad y habitus ambulante. Sociedad y Economía, (31), 15-32.

García-Jerez, A. \& Peralta, M. (2014). Urbanizaciones cerradas y su vinculación con el espacio exterior de la ciudad de Cali: una propuesta metodológica para su análisis. Prospectiva. Revista de trabajo social e intervención social, (19), 197-221.

Gehl, J. (2011). Life between buildings: Using public space (6 ed.). Washington, US: Island Press.

Gehl, J. (2013). Cities for people. Washington, US: Island Press.

Gehl, J., Gemzoe, L., Kirknaes, S. \& Sondergaard, B. (2006). New City Life. Copenhague, Denmark: The Danish Architectural Press.

Gehl, J. \& Svarre, B. (2013). How to study public life. Washington, US: Island Press.

Goffman, E. (1979). Relaciones en público. Microestudios del orden público. Madrid, España: Alianza.

Harvey, D. (2013). Rebel Cities: from the right to the city to the urban revolution. New York, US: Verso books. 
Hernández-García, J. (2013). The production of informal urban space: The barrios of Bogota. In P. Kellett \& J. Hernández-García (Eds.), Researching the Contemporary City. Identity, Environment and Social Inclusion in Developing Urban Areas (pp. 151168). Bogotá, Colombia: Editorial Pontificia Universidad Javeriana.

Hillier, B. (2004). Can Streets be made safe? Urban Design International, 9(1), 31-45.

Hillier, B. \& Hanson, J. (1984). The social logic of space. Cambridge, UK: Cambridge University Press.

Jacobs, J. (1961). The Death and Life of Great American Cities. New York, US: Vintage.

Jeffrey, R. Crime Prevention through environmental design (2 ed.). California, US: SAGE.

Jennings, V., Larson, L. \& Yun, J. (2016). Advancing Sustainability through urban green space: Determinants of health. International Journal of environmental research and public health, 13(2), 196-211.

Jorgensen, L., Ellis, G. \& Ruddell, E. (2012). Fear Perceptions in Public Parks: Interactions of Environmental Concealment, the presence of people recreating, and Gender. Environment and Behavior, 45(7), 803-820.

Lefebvre, H. (1974). The production of space. Oxford, UK: Blackwell.

López de Lucio, R. (2000). El espacio público en la ciudad europea: entre la crisis y la iniciativa de recuperación. Implicaciones para Latinoamérica. Revista Occidente, (230-231), 105-121.

Madanipour, A. (2003). Public and private spaces of the City. New York, US: Routledge.

Mitchel, D. (2003). The right to the city: social justice and the fight for public space. New York, US: Guilford.

Newman, O. (1973). Defensible Space Crime Prevention through Urban Design. New York, US: Collier.

Newman, O. (1975). Reactions to the 'Defensible Space' study and some further findings. International journal of mental health, 4(3), 48-70.

Newman, O. (1995). Defensible Space: A new physical planning tool for urban revitalization. Journal of the American planning association, 61(2), 149-155.

Newman, O. \& Franck, K. (1982). The effects of building size on personal crime and fear of crime. Population and environment, 5(4), 203-220.

Nochian, A., Mohd, O., Maulan, S. \& Rakhshandehroo, M. (2015). A Comprehensive Public Open Space Categorization using Classification System for Sustainable Development of open Spaces. Alam Cipta, 8(1), 29-40. 
Osgood, C. E., Tannenbaum, P. H. \& Suci, G. J. (1957). The measurement of meaning. Illinois, US: University of Illinois Press.

Oviedo, E., Rodríguez , A. \& Rodríguez, P. (2008). Cohesión social/ miedos y políticas de ciudad. URVIO Revista Latinoamericana de Seguridad Ciudadana, (4), 111-120.

Peters, K., Elands, B. \& Buijs, A. (2010). Social interactions in urban parks: Stimulating Social Cohesion. Urban forestry \& Urban greening, 9(2), 93-100.

Porta, S. \& Renne, J. L. (2005). Linking urban design to sustainability: formal indicators of social urban sustainability field research in Perth, Western Australia. Urban Design International, 10(1), 51-64.

Project for Public Spaces. (2000). How to Turn a Place Around: A Handbook for Creating Successful Public Spaces. New York, US: Project for Public Spaces, Inc.

Pyszczek, O. (2012). Los espacios subjetivos del miedo: construcción de la estigmatización espacial en relación con la inseguridad delictiva urbana . Cuadernos de geografía, 21(1), 41-54.

Rau, M. (2005). Prevención del crimen mediante el diseño ambiental en Latinoamérica. Un llamado de acción ambiental comunitaria. En L. Dammert \& G. Paulsen, Ciudad y Seguridad en América Latina (pp. 85-100). Santiago, Chile: Flacso-Chile.

Rengert, G. (1980). Spatial aspects of criminal behavior. In D. E. Georges-Abeyie \& K. D. Harries, Crime: A spatial perspective (pp. 47-57). New York, US: Columbia University Press.

Sampson, R. \& Raudenbush, S. W. (2004). Seeing Disorder: Neighborhood Stigma and the Social Construction of "Broken Windows". Social psychology quarterly, 67(4), 319-342.

Segura, R. (2009). Paisajes del miedo en la ciudad. Miedo y ciudadanía en el espacio urbano de la ciudad de la Plata. Cuaderno Urbano. Espacio, Cultura y Sociedad, $8(8), 59-91$.

Tulloch, M. (2000). The meaning of age difference in the fear of crime. British journal of criminology, 40(3), 451-467.

UN-Habitat. (2007). Enhancing Urban Safety and Security. New York, US: UN-Habitat.

UN-Habitat. (2015a). Public Space. Nueva York, US: UN-Habitat.

UN-Habitat. (2015b). Safer Cities. Nueva York, US: UN-Habitat.

Villanueva, K., Badland, H., Hooper, P., Koohsari, M., Mavoa, S., Davern, M., . . . Goldfeld, S. (2015). Developing indicators of public open space to promote health and wellbeing in communities. Applied geography, (57), 112-119.

Virilio, P. (2012). The administration of fear. Series 10. Cambridge, UK: Semiotext(e). 
Webb, E., Campbell, D., Schwartz, R. \& Sechrest, L. (1996). Unobstrusive Measures Nonreactive research in the social sciences. Chicago, US: Rand Mc Nally.

Whyte, W. (1980). The Social Life of Small Urban Spaces. New York, US: Projects for Public Space.

Wood, L., Frank, L. D. \& Giles-Corti, B. (2010). Sense of community and its relationship with walking and neighborhood design. Social Science \& Medicine, 70(9), 13811390. 